\title{
Decay of Superflow Confined in Thin Torus: A Realization of Tunneling Quantum Fields
}

\author{
M. Nishida and S. Kurihara \\ Department of Physics, Waseda University, 3-Okubo, Shinjuku-ku, Tokyo 169-8555, Japan
}

(September 19, 2018)

\begin{abstract}
The quantum nucleation of phase slips in neutral superfluids confined in a thin torus is investigated by means of the collective coordinate method. We have devised, with numerical justification, a certain collective coordinate to describe the quantum nucleation process of a phase slip. Considering the quantum fluctuation around the local minimum of the action, we calculate the effective mass of the phase slip. Due to the coherence of the condensate throughout the torus, the effective mass is proportional to the circumference $L$ of the torus, and the decay rate has a strong exponential $L$-dependence.
\end{abstract}

PACS numbers: 67.40.Hf, 64.60.Qb, 67.40.Fd

The circulation of superflow in a macroscopic torus container is quantized. However, it has been pointed out that the quantized circulation can decay via the thermal nucleation of phase slips in the case of very narrow torus, or extremely thin superconducting wires 냅. If. If the temperature is low enough, it is probable that the nucleation due to the macroscopic quantum tunneling become dominant [5, 6]. There have been several experiments of superconducting wires which, in fact, indicate the existence of a crossover between the thermal activation behavior in the high temperature regime and the quantum tunneling behavior in the low temperature regime [7]. We expect that similar experiments on the neutral Bose gas systems will become available, in the near future, with the trapped atomic gases [8], or with the liquid ${ }^{4} \mathrm{He}$ in meso-porous materials [9]. The purpose of this Letter is to estimate the probability of the quantum nucleation of phase slips in dilute Bose gas systems.

At sufficiently low temperatures, a condensate in a dilute Bose gas is described by a macroscopic wave function $\psi(\boldsymbol{r})$. We consider a very thin torus container. The circumference $L$ is much larger than the healing length $\xi=\frac{\hbar}{\sqrt{m \rho_{0} g}}$, where $m$ is the mass of an individual atom, $\rho_{0}$ is the density of the condensate (without flow), and $g=\frac{4 \pi \hbar^{2} a}{m}$ is the interatomic interaction, with $a$ being the s-wave scattering length. The cross-sectional area $\sigma$ is much smaller than $\xi^{2}$. We assume that the spatial dependence of $\psi$ in the cross section can be neglected by using the effective number density. We will discuss this point later. Taking $x$ axis along the circumference of the container, the action $S[\psi]$ and the free energy $F[\psi]$ have the Gross-Pitaevskii (GP) form [10]:

$$
\begin{gathered}
S[\psi]=\int d t\left\{\sigma \int_{-\frac{L}{2}}^{\frac{L}{2}} d x \bar{\psi}\left(\mathrm{i} \hbar \frac{\partial}{\partial t}\right) \psi-F[\psi]\right\}, \\
F[\psi]=\sigma \int_{-\frac{L}{2}}^{\frac{L}{2}} d x\left\{\frac{\hbar^{2}}{2 m}\left|\frac{\partial \psi}{\partial x}\right|^{2}+\frac{g}{2}\left(|\psi|^{2}-\frac{\mu}{g}\right)^{2}\right\},
\end{gathered}
$$

where $\mu \sim \rho_{0} g$ is the chemical potential.
If we assume that the effective inertial mass of the phase slip is not greatly dependent on the tunneling path, the most probable tunneling path will be through the valley, passing through two local minima and one saddle point in the functional space of $\psi$. The wave function $\psi$ is determined so as to minimize the free energy functional at each stage of the tunneling process. We calculated the minimum value of the GP free energy numerically with the constraint condition that the average velocity of the superflow had each constant value. We adopt the units in which the unit of length is the healing length $\xi$, the unit of velocity is the sound velocity $c=\sqrt{\frac{\rho_{0} g}{m}}$, and the unit of number density is the density of the condensate $\rho_{0}$. A dimensionless parameter, $\alpha \equiv \rho_{0} \sigma \xi$, determines the importance of quantum fluctuations [5]. We assume $\alpha \gg 1$, and use WKB approximation. Considering $\sigma \ll$ $\xi^{2}, \alpha \ll \rho_{0} \xi^{3} \propto \rho_{0}^{-1 / 2}$, so $\alpha$ is large when the density is low. We include the following term into the free energy Eq. (2):

$$
\lambda\left[\int_{-\frac{L}{2}}^{\frac{L}{2}} d x \rho(x)\left\{\frac{d \theta(x)}{d x}-v_{\mathrm{s}}\right\}\right]^{2},
$$

where $\rho(x)$ and $\theta(x)$ are the density and the phase of the condensate respectively, $v_{\mathrm{s}}$ is the constraint value of the superflow velocity, and $\lambda$ is supposed to take sufficiently large value which is determined by the condition that the difference between the constraint value $v_{\mathrm{s}}$ and the average velocity of the obtained solution be smaller than, say, $1.0 \times 10^{-6} c$. We had $L=20 \xi$ in a mesh of 100 points, and used the simulated annealing method [11]. Because there are a lot of local minima which correspond to multiple phase slip solutions, it is difficult to obtain true minimum values with other methods. In order to avoid being trapped in these local minima, we introduce random noises which correspond to thermal fluctuations at given temperature. The calculation proceeds gradually from high temperature region to low temperature region, and continues until equilibrium values are reached. In our calculation, the final temperature is $1.5 \times 10^{-10} \rho_{0} g$, and the expected error of the free energy is $O\left(10^{-4} \rho_{0} g\right)$. 
Extrema of the GP free energy functional are solutions to the time-independent GP equation:

$$
-\frac{1}{2} \frac{\partial^{2} \psi}{\partial x^{2}}+\left(|\psi|^{2}-1\right) \psi=0 .
$$

There are two kinds of solutions to this equation [3]. The first kind of solution corresponds to a free-energy local minimum:

$$
\psi_{\mathrm{m}}=\mathrm{i} \sqrt{1-\frac{\kappa^{2}}{2}} e^{\mathrm{i} \kappa x}
$$

where $\kappa$ is the phase gradient or, in other words, the superflow velocity. The second kind corresponds to a free-energy saddle point:

$$
\psi_{\mathrm{s}}=e^{\mathrm{i} \kappa x}\left\{\sqrt{1-\frac{3}{2} \kappa^{2}} \tanh \left(\sqrt{1-\frac{3}{2} \kappa^{2}} x\right)-\mathrm{i} \kappa\right\} .
$$

The phase gradient of $\psi_{\mathrm{s}}$ approaches $\kappa$ asymptotically as we go away from the phase slip center $(x=0)$. Hereafter, we call $\kappa$ "asymptotic velocity". If we can find the function which interpolates these two kinds of solutions smoothly, and fits the numerical results, we may use it as the wave function which describes the condensate during the whole nucleation process. Considering the periodic boundary condition, we have found the following interpolation function:

$$
\begin{aligned}
\psi_{\mathrm{i}}= & \sqrt{1-\frac{\kappa^{2}}{2}} e^{\mathrm{i} \kappa x} \\
& \times\left\{\sin \frac{q}{2} \tanh \left(\sqrt{1-\frac{\kappa^{2}}{2}} \sin \frac{q}{2} x\right)+\mathrm{i} \cos \frac{q}{2}\right\} .
\end{aligned}
$$

We must determine the parameter $q$ so that $\psi_{\text {i }}$ satisfies the periodic boundary condition. If $q L \gg 1$, we can take the form,

$$
q=\kappa L-2 \pi n,
$$

where $n$ is the winding number of the phase around the circumference of the container $(n=0, \pm 1, \pm 2, \ldots)$. The parameter $q$ expresses the phase difference between two sides of the phase slip center. Hereafter, we call it "phase jump". We can adopt either asymptotic velocity or phase jump as an appropriate collective coordinate to describe the nucleation process. Substituting Eq. (7) into Eq. (2), the potential energy for the asymptotic velocity $\kappa$ is obtained as follows:

$$
\begin{aligned}
V(\kappa) & =\frac{4}{3}\left(1-\frac{\kappa^{2}}{2}\right)^{\frac{3}{2}} \sin ^{3} \frac{q}{2} \\
& -2 \kappa\left(1-\frac{\kappa^{2}}{2}\right) \sin \frac{q}{2} \cos \frac{q}{2}+\frac{L}{2}\left(\kappa^{2}-\frac{\kappa^{4}}{4}\right) .
\end{aligned}
$$

Using $\kappa$ as a fitting parameter, we fit the amplitude and phase of the wave function Eq. (7) with those which are given by the numerical minimization. The phase jump changes about $L(=20) \%$ when $\kappa$ changes $1 \%$ (see Eq. (8)), so the shape of the phase is very sensitive to the value of $\kappa$. Fig. (1) shows that Eq. (9) is in good agreement with the numerical results.

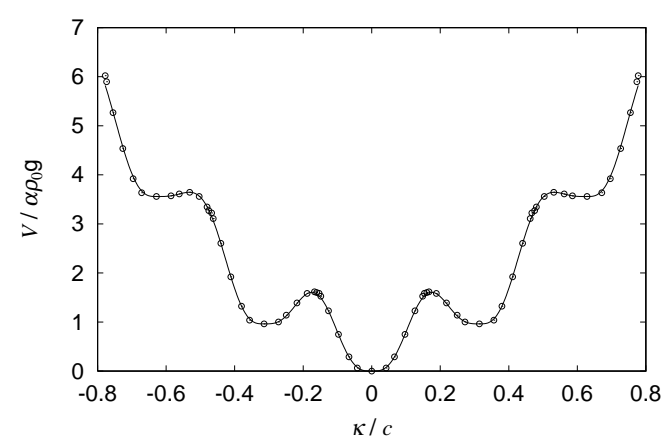

FIG. 1. Potential energy for the collective coordinate $\kappa$. The points are the minimum values of the GP free energy which were calculated numerically. The solid line denotes the value of the potential energy Eq. (9) which is calculated using the interpolation wave function Eq. (7).

We then consider the effective inertial mass for the motion of the collective coordinate. Because of the translational invariance, the total momentum must be conserved between the initial state and the final state. If there were no contributions from the noncondensed component, the condensate would not lose the momentum and stay at the local minimum. However, quantum fluctuations of $\psi$ exist around the distorted condensate wave function $\psi_{\mathrm{i}}$, and these fluctuations will play the role of a noncondensed component. Therefore, the condensate can oscillate around the local minimum and tunnel to another local minimum by exchanging the momentum with quantum fluctuations, even at zero temperature. The frequency of the zero-point oscillation of the collective coordinates around the local minimum will have the same value of the collective mode whose wave length is $L$. Because we have already determined the potential energy, Eq. (9), we can estimate the effective inertial mass from the frequency of the collective mode and the curvature of the potential energy for $\kappa$ or $q$ at the local minimum. In this procedure, we assume that the inertial mass is not greatly dependent on the collective coordinate, and approximate it to the value at local minimum. The coupling with the collective modes also suggests that there are the effects of dissipation, which will suppress the tunneling probability $\mid 12$. However, we will not take into account these effects, but concentrate our interests on the bounce motion of $\kappa$ or $q$. In order to clarify these two points, we would have to consider quantum fluctuations explicitly and integrate them out, thus requiring further study.

In order to estimate the frequency of the collective mode, we consider the fluctuation around the local min- 
imum solution, Eq. (5). We substitute $\psi=\psi_{\mathrm{m}}+(u+$ $\mathrm{i} v) \mathrm{e}^{\mathrm{i} \kappa x}$ into the action, Eq. (1), and retain the terms up to the second order of $u, v$, then we have

$$
\begin{aligned}
S[u, v]= & \hbar \alpha \int d t\left[-V(\kappa)+\int d x\left\{2 i v\left(\frac{\partial}{\partial t} \sqrt{1-\frac{\kappa^{2}}{2}}\right)\right.\right. \\
& \left.\left.+\frac{1}{2}\left(\begin{array}{ll}
u & v
\end{array}\right) \boldsymbol{G}^{-1}\left(\begin{array}{c}
u \\
v
\end{array}\right)\right\}\right],
\end{aligned}
$$

where $\boldsymbol{G}$ is the Green's function for $u, v$, whose Fourier component is given by

$$
\begin{aligned}
\boldsymbol{G}= & \left\{\frac{k^{2}}{2}\left(\frac{k^{2}}{2}+2\left(1-\frac{\kappa^{2}}{2}\right)\right)-(\kappa k-\omega)^{2}\right\}^{-1} \\
& \times \frac{1}{2}\left(\begin{array}{cc}
-\frac{k^{2}}{2}-2\left(1-\frac{\kappa^{2}}{2}\right) \mathrm{i}(\kappa k-\omega) \\
-\mathrm{i}(\kappa k-\omega) & -\frac{k^{2}}{2}
\end{array}\right) .
\end{aligned}
$$

$\boldsymbol{G}$ has the poles which correspond to the Bogoliubov modes in the flowing medium. We can derive these poles by Galilei transformation from the co-flowing frame to the lab frame, noting that the density is now $1-\frac{\kappa^{2}}{2}$. From these poles, we estimate the effective angular frequency of the oscillation of $\kappa$ by the following relation:

$$
\begin{aligned}
& \omega_{\text {eff }}^{2}-\omega^{2} \\
& =\left.\left[\frac{k^{2}}{2}\left\{\frac{k^{2}}{2}+2\left(1-\frac{\kappa^{2}}{2}\right)\right\}-(\kappa k-\omega)^{2}\right]\right|_{k=\frac{2 \pi}{L}} .
\end{aligned}
$$

Around the $n$th local minimum, whose velocity is $\kappa_{n}=$ $\frac{2 \pi n}{L}$, the effective angular frequency becomes

$$
\omega_{\mathrm{eff}}^{2}(n)=\left(\frac{2 \pi}{L}\right)^{2}\left\{1-\frac{3}{2} \kappa_{n}^{2}+\left(\frac{\pi}{L}\right)^{2}\right\} .
$$

When $q$ is so small that we cannot assume $q L \gg 1$, Eq. (8) and Eq. (9) become inaccurate and we must determine the relationship between $q$ and $\kappa$ with direct consideration of the periodic boundary condition. After doing so, the potential curvature at the $n$th local minimum becomes

$$
V^{\prime \prime}\left(\kappa_{n}\right)=L\left(1-\frac{3}{2} \kappa_{n}^{2}\right),
$$

where the prime denotes differentiation with respect to $\kappa$.

From Eq. (13) and Eq. (14), the effective inertial mass for $\kappa$ is given by

$$
\begin{aligned}
M_{\kappa}(n) & =\frac{V^{\prime \prime}\left(\kappa_{n}\right)}{\omega_{\mathrm{eff}}^{2}(n)}=\frac{L^{3}}{(2 \pi)^{2}}\left(\frac{1-\frac{3}{2} \kappa_{n}^{2}}{1-\frac{3}{2} \kappa_{n}^{2}+\left(\frac{\pi}{L}\right)^{2}}\right) \\
& \sim \frac{L^{3}}{(2 \pi)^{2}} \quad(L \gg 1) .
\end{aligned}
$$

We now consider the tunneling problem from $n$th local minimum to the $(n-1)$ th local minimum. The tunneling rate $\Gamma$ is given by $\Gamma=A e^{-S_{\mathrm{B}} / \hbar}$, where $S_{\mathrm{B}}$ is the bounce action, which is the Euclidean action for the bounce motion of $\kappa[13]$ :

$$
S_{\mathrm{B}}=\hbar \alpha \int d \tau\left\{\frac{M_{\kappa}(n)}{2} \dot{\kappa}^{2}+V(\kappa)-V\left(\kappa_{n}\right)\right\} .
$$

Because $\kappa$ contains either the winding number $n$ or the system length $L$, it is more convenient to use the phase jump $q$ for the collective coordinate when we consider the dependence of the tunneling rate $\Gamma$ on the superflow velocity $\kappa_{n}$ or the system length $L$. Rewriting the bounce action $S_{\mathrm{B}}$ by using the phase jump $q=\left(\kappa-\kappa_{n-1}\right) L$, we obtain

$$
S_{\mathrm{B}}=2 \hbar \alpha \int_{q_{0}}^{2 \pi} d q \sqrt{2 M_{q}(n) V_{n}(q)},
$$

where $q_{0}$ is the classical turning point of $-V_{n}(q)$,

$$
\begin{gathered}
M_{q}(n)=\frac{M_{\kappa}(n)}{L^{2}} \sim \frac{L}{(2 \pi)^{2}} \quad(L \gg 1), \\
V_{n}(q)=V\left(q / L+\kappa_{n-1}\right)-V\left(\kappa_{n}\right) \\
\sim \frac{4}{3}\left(1-\frac{\kappa_{n}^{2}}{2}\right)^{\frac{3}{2}} \sin ^{3} \frac{q}{2}-\kappa_{n}\left(1-\frac{\kappa_{n}^{2}}{2}\right) \sin q \\
(L \gg 1) .
\end{gathered}
$$

We thus find that the effective inertial mass for the nucleation of a phase slip is proportional to the system length $L$. It basically comes from the fact that the potential curvature is proportional to the system length, (see Eq. (14)). When a phase slip nucleates, the whole wave function of the condensate changes its configuration due to its overall coherence, so the free energy change becomes larger as the system circumference becomes longer. Therefore the coherence of the condensate wave function is the origin of the $L$-dependence of the inertial mass.

We can calculate the bounce action analytically in the zero-current limit $\left(\kappa_{n} \rightarrow 0\right)$ :

$$
\begin{aligned}
S_{0} & =\frac{2 \sqrt{2} \hbar \alpha \sqrt{L}}{\sqrt{3} \pi} \int_{0}^{2 \pi} d q \sin ^{\frac{3}{2}} \frac{q}{2} \\
& \sim 1.82 \hbar \alpha \sqrt{L} .
\end{aligned}
$$

Fig. 2 shows the bounce actions normalized by $S_{0}$ in the five different cases with the circumferences of $50-10000 \xi$. In all cases, the ratio $S_{\mathrm{B}} / S_{0}$ goes to zero at the common velocity $v_{\mathrm{c}} \sim \sqrt{2 / 3} c$. This velocity corresponds to the critical velocity of the superflow. If the asymptotic velocity $\kappa$ goes beyond $v_{\mathrm{c}}$, the saddle point solution to the time-independent GP equation, Eq. (6), will vanish. No stable local minima exist for velocity $\kappa_{n}>v_{\mathrm{c}}$. The value of $v_{\mathrm{c}}$ agrees with the one which is derived by Landau's criterion. As $\kappa_{n}$ approaches the value 


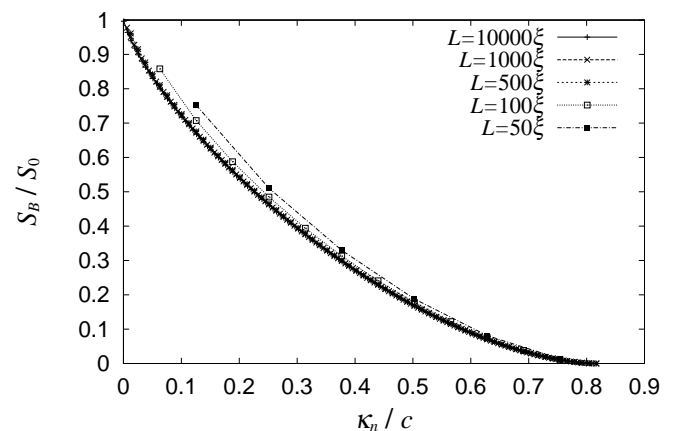

FIG. 2. Bounce actions normalized by $S_{0}$, which are calculated for the five different samples with the circumferences of $50-10000 \xi$.

of $v_{\mathrm{c}}$, the bounce action becomes extremely small. In the case of $L=100 \xi, n=12$, the normalized bounce action $S_{\mathrm{B}} / S_{0}$ becomes 0.012 .

We see from Fig. 2 that there is no sizable $L$ dependence for $L \gg 1$ under consideration. If $L$ is larger than $100 \xi$, all the data seem to be on the same line. Therefore we conclude that the $L$-dependence mainly comes from the effective inertial mass, and the bounce action is proportional to $L^{\frac{1}{2}}$. This situation is completely different from that in the thermal nucleation processes. In the thermal nucleation processes, the decay rate is estimated by the Arrhenius formula $\Gamma \propto e^{-\delta F / k_{\mathrm{B}} T}$, which is determined only by the barrier height $\delta F$, so there is no dependence of the decay rate on the system length 迎田. In the quantum nucleation, the coherence of the condensate wave function becomes important, and the decay rate has the strong $L$-dependence:

$$
\Gamma \propto \exp \left\{-1.82 \alpha \sqrt{L} f\left(\kappa_{n}\right)\right\},
$$

where $f\left(\kappa_{n}\right)$ is the function which depends only on the superflow velocity, and whose shape is shown in Fig. 2 .

We now discuss the spatial dependence of $\psi$ in the cross section. If we consider a complete set of radial eigenfunctions, the energy scale of the $\nu$ th eigenstate will become $\frac{\hbar^{2} \nu^{2}}{m \sigma}$. Because it is much larger than $\mu \sim \frac{\hbar^{2}}{m \xi^{2}}$ in our model when $\nu \neq 0$, the $\nu=0$ channel will become dominant 顿. Besides, the energy scale of the potential barrier is $\alpha \mu \sim \frac{\hbar^{2} \sigma \rho_{0}}{m \xi}$, which corresponds to the energy of $\frac{\rho_{0} \sigma^{2}}{\xi \nu^{2}}$ particles excited into the $\nu$ th channel. The ratio to the total number is $\frac{\sigma}{\xi L \nu^{2}} \ll 1$ when $\nu \neq 0$. These estimations seem to indicate that we can safely neglect the spatial dependence in the cross section. However, there are other effects to be considered: For example, the nonlinearity of GP equation. It will cause couplings between the current direction and the radial direction. If we take this into account, we may find a more appropriate tunneling path. Moreover, when the condensate is squeezed in a region whose size is smaller than the healing length, the phase of the condensate will fluctuate intensively. Because phase fluctuations $\left\langle(\delta \theta)^{2}\right\rangle$ will effectively suppress the amplitude of the wave function by the factor $1-\frac{1}{2}\left\langle(\delta \theta)^{2}\right\rangle+O\left((\delta \theta)^{4}\right)$. This means that the effective number density of the condensate, and thus the parameter $\alpha$, which gives the scale of bounce action, will become smaller than the results of the present calculation. Therefore, it is probable that our result of tunneling rate is an underestimate. The analysis of these effects is left for the future work.

In conclusion, we have studied the quantum nucleation of phase slips in torus shaped superfluids by means of the collective coordinate method. We have found a wave function which interpolates the local minimum and saddle point solutions to the time-independent GP equation, and this fits our numerical results. We have determined the appropriate collective coordinate which describes the whole nucleation process, and obtained the analytic form of the potential barrier. We have shown that the effective inertial mass is proportional to the system length $L$ due to the coherence of the condensate. Because the length dependence of the bounce action mainly comes from the mass, we have concluded that the bounce action is proportional to $L^{\frac{1}{2}}$.

We thank T. Inoue, I. Tomita, L. Mowrey, and T. Minoguchi for useful discussion. This work is supported by Grant-in-Aid for Scientific Research from the Ministry of Education, Science, Sports, and Culture of Japan.

[1] W. A. Little, Phys. Rev. 156, 396 (1967).

[2] J. S. Langer and V. Ambegaokar, Phys. Rev. 164, 498 (1967).

[3] D. E. McCumber and B. L. Halperin, Phys. Rev. B 1, 1054 (1970).

[4] E. J. Mueller, P. M. Goldbart, and Y. Lyanda-Geller, Phys. Rev. A 57, R1505 (1998).

[5] J. A. Freire, D. P. Arovas, and H. Levine, Phys. Rev. Lett. 79, 5054 (1997).

[6] S. Saito and Y. Murayama, Phys. Lett. A 135, 55 (1989).

[7] N. Giordano, Physica B 203, 460 (1994) and refs. therin.

[8] M. H. Anderson et al., Science 269, 198 (1996); K. B. Davis et al., Phys. Rev. Lett. 75, 3969 (1995).

[9] K. Shirahama et al., Phys. Rev. Lett. 64, 1541 (1990).

[10] E. P. Gross, Nuovo Cimento 20, 454 (1961); J. Math. Phys. 4, 195 (1963); L. P. Pitaevskii, Zh. Eksp. Teor. Fiz. 40, 646 (1961) [Sov. Phys. JETP 13, 451 (1961)].

[11] W. H. Press et al., Numerical Recipes (Cambridge University Press, Cambridge, England, 1986).

[12] A. O. Caldeira and A. J. Leggett, Phys. Rev. Lett. 46, 211 (1981).

[13] S. Coleman, Phys. Rev. D 15, 2929 (1977). 